\title{
掺铋钇铁石榴石磁光陶瓷的热压烧结及其性能研究
}

\author{
邹 顺 ${ }^{1,2}$, 何夕云 ${ }^{1}$, 曾 霞 $^{1}$, 仇萍蒜 ${ }^{1}$, 凌 亮 $^{1}$, 孙大志 ${ }^{2}$ \\ (1. 中国科学院 上海硅酸盐研究所, 上海 200050; 2. 上海师范大学 化学与材料科学学院, 上海 200234)
}

摘 要: 钎铁石榴石 $\left(\mathrm{Y}_{3} \mathrm{Fe}_{5} \mathrm{O}_{12}\right.$, YIG) 材料因其优异的磁性能和磁光性能在微波通信、激光技术和光纤通讯等领域具 有重要应用。离子掺杂是提高 YIG 材料磁光性能的有效途径之一, 本研究选择离子半径适配的 $\mathrm{Bi}^{3+}$ 掺杂改性 YIG 陶瓷以提高材料的磁光性能。本工作采用固相法热压烧结制备 $\mathrm{Bi}_{x} \mathrm{Y}_{3-x} \mathrm{Fe}_{5} \mathrm{O}_{12}(x=0 、 0.3 、 0.6 、 0.9)$ 陶瓷, 并研究 $\mathrm{Bi}^{3+}$ 掺杂对 YIG 陶瓷材料相结构、微观形貌、红外透过性、磁性能以及磁光性能的影响。结果表明: 陶瓷样品均呈石 榴石立方相结构; 显微结构致密, $\mathrm{Bi}^{3+}$ 掺杂后晶粒尺寸不同程度增大; 样品红外透过率良好, 随 $\mathrm{Bi}^{3+}$ 掺杂量增大而 降低; 陶瓷样品的法拉第旋转角随 $\mathrm{Bi}^{3+}$ 掺杂量增加呈线性变化, $\mathrm{Bi}^{3+}$ 掺杂量每增加 $1 \%$ (原子分数), 在波长 $1064 \mathrm{~nm}$ 和 $1550 \mathrm{~nm}$ 处变化量分别约为 $-49.0\left({ }^{\circ}\right) / \mathrm{cm}$ 和 $-30.2\left(^{\circ}\right) / \mathrm{cm} 。 \mathrm{Bi}_{0.6} \mathrm{Y}_{2.4} \mathrm{Fe}_{5} \mathrm{O}_{12}$ 陶瓷样品在 $1064 \mathrm{~nm}$ 和 $1550 \mathrm{~nm}$ 波长下法 拉第旋转角分别达到 $-703.3\left({ }^{\circ}\right) / \mathrm{cm}$ 和 $-461.5\left(^{\circ}\right) / \mathrm{cm}$, 绝对值远高于未掺杂 YIG 陶瓷的 $277.6\left({ }^{\circ}\right) / \mathrm{cm}$ 和 $172.0\left({ }^{\circ}\right) / \mathrm{cm}$ 。 由此可见，掺杂适量 $\mathrm{Bi}^{3+}$ 可以显著增强 YIG 陶瓷材料的磁光性能。

关 键 词: 钎铁石榴石; 磁光陶瓷; 铋掺杂; 红外透光率; 法拉第旋转

中图分类号: TM284 文献标志码: A

\section{Microstructure and Properties of Bi-doped Yttrium Iron Garnet Magneto-optical Ceramics Prepared by Hot-pressing Sintering Process}

\author{
ZOU Shun ${ }^{1,2}$, HE Xiyun ${ }^{1}$, ZENG Xia ${ }^{1}$, QIU Pingsun ${ }^{1}$, LING Liang ${ }^{1}$, SUN Dazhi ${ }^{2}$ \\ (1. Shanghai Institute of Ceramics, Chinese Academy of Sciences, Shanghai 200050, China; 2. College of Chemistry and \\ Materials Science, Shanghai Normal University, Shanghai 200234, China)
}

\begin{abstract}
Yttrium iron garnet $\left(\mathrm{Y}_{3} \mathrm{Fe}_{5} \mathrm{O}_{12}, \mathrm{YIG}\right)$ materials with excellent magnetic and magneto-optical performances have been widely used in various fields such as the microwave communication, laser technology and optical fiber communication. Ion doping is considered as an effective method to improve the magneto-optical performance of YIG materials. In this study, $\mathrm{Bi}^{3+}$ with appropriate ion radius were selected to modify YIG ceramics to improve the magneto-optical properties of the materials. A series of $\mathrm{Bi}_{x} \mathrm{Y}_{3-x} \mathrm{Fe}_{5} \mathrm{O}_{12}(x=0,0.3,0.6,0.9)$ ceramics were prepared by the hot-pressing sintering process with the solid-state reaction. Phase structure, micro-morphology, infrared transmittance, magnetic and magneto-optical properties of the sintered $\mathrm{Bi}_{x} \mathrm{Y}_{3-x} \mathrm{Fe}_{5} \mathrm{O}_{12}(x=0,0.3,0.6,0.9)$ ceramics were investigated. All sintered $\mathrm{Bi}^{3+}$-doped YIG ceramic samples exhibit the pure garnet phase structure with dense microstructure, and the grain size increases with $\mathrm{Bi}^{3+}$ doping. The infrared transmittance of the sample is good, but it decreases with the increase of $\mathrm{Bi}^{3+}$ doping content. The Faraday rotation angle of the YIG ceramic sample changes linearly with the $\mathrm{Bi}^{3+}$ content increase. When the $\mathrm{Bi}^{3+}$ content increases about $1 \%$ (in atom), Faraday rotation angle
\end{abstract}

收稿日期: 2021-09-30; 收到修改稿日期：2021-11-16; 网络出版日期：2021-12-24

作者简介: 邹顺(1995-), 男, 硕士研究生. E-mail: shunzou950105@163.com ZOU Shun (1995-), male, Master candidate.E-mail: shunzou950105@163.com

通信作者：何夕云，研究员. E-mail: xyhe@mail.sic.ac.cn; 孙大志, 教授. E-mail: sundazhi@shnu.edu.cn HE Xiyun, professor. E-mail: xyhe@mail.sic.ac.cn; SUN Dazhi, professor E-mail: sundazhi@shnu.edu.cn 
changes about $-49.0\left(^{\circ}\right) / \mathrm{cm}$ and $-30.2\left(^{\circ}\right) / \mathrm{cm}$ at $1064 \mathrm{~nm}$ and $1550 \mathrm{~nm}$ wavelengths, respectively. Faraday rotation angles of $\mathrm{Bi}_{0.6} \mathrm{Y}_{2.4} \mathrm{Fe}_{5} \mathrm{O}_{12}$ ceramic sample at wavelengths of $1064 \mathrm{~nm}$ and $1550 \mathrm{~nm}$ are $-703.3\left({ }^{\circ}\right) / \mathrm{cm}$ and $-461.5\left(^{\circ}\right) / \mathrm{cm}$, respectively, which are much higher than that of the undoped YIG ceramics $\left(277.6\left({ }^{\circ}\right) / \mathrm{cm}\right.$ and $172.0\left(^{\circ}\right) / \mathrm{cm}$, respectively). The magneto-optical performance of YIG ceramics is enhanced obviously by the $\mathrm{Bi}^{3+}$ doping.

Key words: yttrium iron garnet; magneto-optical ceramics; Bi-doped; infrared transmittance; Faraday rotation

法拉第效应是磁光材料重要磁光特性之一, 表 现为当磁场作用于磁光材料时, 线性偏振光以平行 于磁场方向通过磁光材料后, 偏振平面发生旋转, 旋转方向仅与磁场方向有关, 与光的传播方向无关, 旋转角度大小与材料厚度及磁场强度有关。磁光材 料应用其法拉第效应可制备光隔离器 ${ }^{[1]}$ 、相位调制 器 ${ }^{[2]}$ 、偏振控制器 ${ }^{[3]}$ 和数据存储器 ${ }^{[4]}$ 等器件。YIG 材 料 ${ }^{[5]}$ 在饱和外磁场下法拉第旋转角大, 且红外透过 率高、光吸收率低, 是制作法拉第旋转器 (Faraday Rotator, FR)、红外光隔离器(Isolator)等器件的重要 核心材料。目前已应用的主要为 YIG 单晶材料, 通 常采用助熔剂法 ${ }^{[6]}$ 、流动溶剂浮区(Traveling Solvent

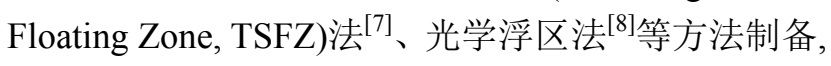
例如上海应用技术大学徐家跃等 ${ }^{[9]}$ 以 $\mathrm{PbO}-\mathrm{B}_{2} \mathrm{O}_{3}$ 为 助熔剂成功生长出高透过率 YIG 单晶。中国科学院 上海硅酸盐研究所武安华等 ${ }^{[10]}$ 采用光学浮区法成 功制备了具有优异磁光性能的 Ce 掺杂的 YIG 晶体。 YIG 非一致熔融性的特点, 单晶制备难以获得大体 积块材，限制了其应用。2018 年日本 Ikesue 等 ${ }^{[11]}$ 首次通过氧气氛无压和 $\mathrm{O}_{2}-\mathrm{Ar}$ 气氛热等静压两步烧 结法获得磁光性能优良的高透过率 YIG 磁光陶瓷, 该报道预示采用陶瓷工艺有望获得组成精确可控、 磁光性能优异、红外透过率高的大尺寸 YIG 材料。

优质的磁光材料需要以尽可能小的尺寸去获得 更大的法拉第旋转角, 即提高磁光材料的旋光率 $\left(\theta_{\mathrm{F}}\right)$ 。立方 $\mathrm{YIG}$ 晶格属于空间群 $\mathrm{Ia} \mathrm{d}^{[12]}$, 由三个磁 性亚晶格组成, 分别为八面体位(a)、四面体位 (d) 和 十二面体位(c)亚晶格 ${ }^{[13]}$, 其中十二面体(c)亚晶格 被非磁性离子 $\mathrm{Y}^{3+}$ 离子占据。前期已有研究通过离 子半径适配的异质离子取代 $\mathrm{Y}^{3+}$, 以增强 $\mathrm{YIG}$ 的磁 光性能, 如三价稀土离子 ${ }^{[14-17]}$ 或 $\mathrm{Bi}^{3+}$ 离子 ${ }^{[18-19]}$ 等。 在 $\mathrm{YIG}$ 材料中, 当 $\mathrm{Bi}^{3+}$ 离子部分取代 $\mathrm{Y}^{3+}$ 离子时, 其 法拉第旋转角出现反向旋转, 随 $\mathrm{Bi}^{3+}$ 掺杂量增加近 似线性变化 ${ }^{[20]}$ 。文献 $[21]$ 认为: $\mathrm{Bi}^{3+}$ 具有相当大的自 旋-轨道耦合, $\mathrm{Bi}^{3+}$ 的 $6 \mathrm{p}$ 轨道能级 $(\sim 1.6 \mathrm{eV})$ 与 $\mathrm{Fe}^{3+}$ 的 $3 \mathrm{~d}$ 轨道能级 $(\sim 1.52 \mathrm{eV})$ 十分接近, 因而 $\mathrm{Bi}^{3+}$ 的 $6 \mathrm{p}$ 轨 道可能与 $\mathrm{O}^{2-}$ 的 $2 \mathrm{p}$ 轨道、 $\mathrm{Fe}^{3+}$ 的 $3 \mathrm{~d}$ 轨道发生简并, 使 得磁光效应相关的激发态能级分裂增大。
目前 $\mathrm{Bi}^{3+}$ 掺杂铁石榴石材料主要是采用 $\mathrm{LPE}$ (液相外延) 法生产单晶材料为主 ${ }^{[22-23]}$, 只能在 $\mathrm{GGG}\left(\mathrm{Gd}_{3} \mathrm{Ga}_{5} \mathrm{O}_{12}\right)$ 基祄底材料上外延生长, 生长速 度慢、生产周期长、成本非常高。Ikesue ${ }^{[11]}$ 在氧气 氛围下热等静压(Hot Isostatic Pressing, HIP)烧结红 外透明的 $\mathrm{Bi}^{3+}$ 掺杂 $\mathrm{YIG}$ 陶瓷, 烧结的 $\mathrm{Bi}_{1.1} \mathrm{Y}_{1.9} \mathrm{Fe}_{5} \mathrm{O}_{12}$ 陶瓷在 $1550 \mathrm{~nm}$ 处法拉第旋转角为 $-970\left(^{\circ}\right) / \mathrm{cm}$, 且 随着 $\mathrm{Bi}^{3+}$ 掺杂每增加 $1 \%$ (原子分数), $\theta_{\mathrm{F}}$ 变化量约 $-31.2\left({ }^{\circ}\right) / \mathrm{cm}$ 。这是首次成功制备出用于磁光隔离器 的 $\mathrm{Bi}^{3+}$ 掺杂 $\mathrm{YIG}$ 陶瓷, 但由于该制备方法对设备要 求高, 技术复杂, 目前还未能产业化应用。若能采用 热压烧结工艺制备 $\mathrm{Bi}^{3+}$ 掺杂铁石榴石多晶陶瓷, 不 仅可以缩短制备周期, 降低生产成本, 且易制备大 体积块材。

本研究以 $\mathrm{Bi}_{2} \mathrm{O}_{3} 、 \mathrm{Y}_{2} \mathrm{O}_{3}$ 和 $\mathrm{Fe}_{2} \mathrm{O}_{3}$ 等氧化物粉体 为原料, 采用热压烧结技术通过固相反应法制备了 $\mathrm{Bi}^{3+}$ 掺杂 $\mathrm{YIG}$ 系列多晶陶瓷。系统考察了烧结陶瓷 样品的物相结构和显微形貌, 采用紫外-可见分光 光度计及傅里叶红外光谱仪(FT-IR)测试抛光后陶 瓷样品的红外透过性, 研究了饱和外磁场下陶瓷样 品的磁光性能。

\section{1 实验方法}

\section{1 原材料及制备}

采用的原料粉体有 $\mathrm{Bi}_{2} \mathrm{O}_{3}$ (国药，99\%)、 $\mathrm{Y}_{2} \mathrm{O}_{3}$ (Aladdin, 99.9\%) 和 $\mathrm{Fe}_{2} \mathrm{O}_{3}$ (Aladdin, 99.9\%)。根 据 $\mathrm{Bi}_{x} \mathrm{Y}_{1-x} \mathrm{Fe}_{5} \mathrm{O}_{12}(x=0 、 0.3 、 0.6 、 0.9)$ 的化学计量比 称量原料, 用乙醇作为研磨介质, 置于装有钢球的 球磨罐中, 在滚筒式球磨机中球磨 $36 \mathrm{~h}$ 。球磨后, 将 粉体混合浆料置于干燥箱中充分烘干。充分干燥后, 将烘干料碾碎研磨成粉末并添加粘结剂(PVA)进行 造粒。将造粒后分散均匀的粉末用轴向压力干压成 $\phi 15 \mathrm{~mm} \times 20 \mathrm{~mm}$ 的圆柱形素坏, 然后在 $220 \mathrm{MPa}$ 下 进行冷等静压(Cold Isostatic Pressing, CIP)处理。最 后, 将样品装入热压炉内, 在空气气氛下轴向热压 $(\sim 20 \mathrm{MPa})$ 烧结。分别在 $1050 、 1150 、 1250$ 和 $1300{ }^{\circ} \mathrm{C}$ 下烧结 4 8 h, 再将烧结好的陶瓷块体进行磨平、切 
片、双面抛光等加工处理，以便于测试表征。

\section{2 样品表征}

采用阿基米德法测量烧结样品的密度。通过 XRD (X-ray diffraction, D/max $2550 \mathrm{~V}$, Japan)鉴定晶 体结构。使用振动样品磁强计 (Lakeshore 7400, America) 获得样品的磁滞回线 $(B-H)$ 。透射率曲线测 试分为两部分: 近红外波段(1.0 2.5 $\mu \mathrm{m})$ 使用紫外可见分光光度计(Cary-5000, America)测量, 中红外 波段 (2.5 10.0 $\mu \mathrm{m})$ 使用傅里叶红外光谱仪(Fourier infrared spectrometer, FT-IR Tensor 27, Germany)测 量。此外, 将抛光后的样品置于 $0.3 \sim 0.35 \mathrm{~T}$ 磁场中 测量法拉第旋转角并计算样品的 $\theta_{\mathrm{F}}$ 。

\section{2 结果与讨论}

\section{1 密度测试}

烧结后的 $\mathrm{Bi}_{x} \mathrm{Y}_{1-x} \mathrm{Fe}_{5} \mathrm{O}_{12}(x=0 、 0.3 、 0.6 、 0.9)$ 陶 瓷样品的密度随烧结温度不同而变化, 见图 1。除掺 杂量为 $x=0$ 的陶瓷样品密度随温度升高而增加, 其 余掺杂量下的陶瓷样品密度随温度升高均呈现先增 加后降低的趋势。对于 $x=0.3 、 0.6 、 0.9$ 的陶瓷样品, 烧结后密度最大的样品对应的烧结温度分别为 1250、1250 和 $1150{ }^{\circ} \mathrm{C}$ 。从实物图中可见，在 $1300{ }^{\circ} \mathrm{C}$ 下烧结除 $x=0$ 的样品未过烧开裂, 其他掺杂浓度的 样品均已经过烧甚至开裂，见图 1(b)。

\section{2 红外光学测试}

图 2 为不同烧结温度 $\mathrm{Bi}_{x} \mathrm{Y}_{1-x} \mathrm{Fe}_{5} \mathrm{O}_{12}$ 陶瓷样品在 1.0 10.0 $\mu \mathrm{m}$ 波长范围内的红外透过率曲线, 测量时 陶瓷样品厚度为 $0.4 \mathrm{~mm}$ 。可以发现, 不同 $\mathrm{Bi}^{3+}$ 掺杂 量 $\mathrm{Bi}_{x} \mathrm{Y}_{1-x} \mathrm{Fe}_{5} \mathrm{O}_{12}$ 陶瓷样品达到最佳透过率所对应的 烧结温度变化很大, $\mathrm{Bi}^{3+}$ 掺杂量 $x=0 、 0.3 、 0.6 、 0.9$

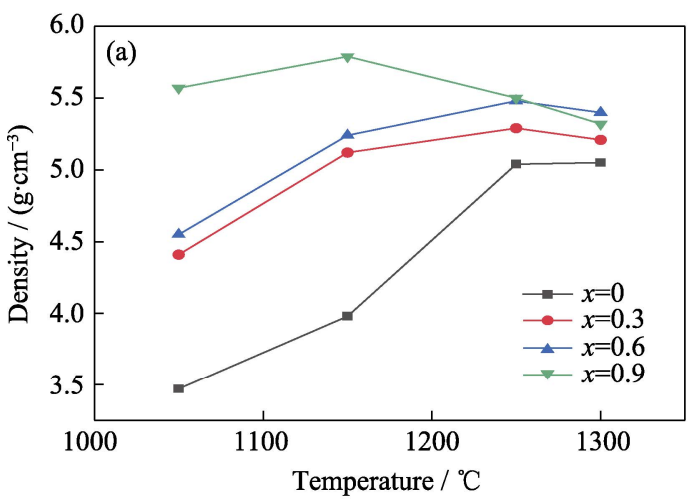

时具有最佳红外透过率的陶瓷样品所对应烧结温度 分别为 $1300 、 1250 、 1150 、 1050{ }^{\circ} \mathrm{C}$ 。而掺杂量 $x=0.6$ 与 0.9 时, 尽管陶瓷样品在密度测试中烧结最致密 时的温度分别为 1250 与 $1150{ }^{\circ} \mathrm{C}$, 但最致密陶瓷样 品所对应烧结温度与透过率最佳陶瓷样品所对应烧 结温度并不相同。如表 1 所示, 对于掺杂量 $x=0.6$ 与 0.9 的 $\mathrm{Bi}_{x} \mathrm{Y}_{1-x} \mathrm{Fe}_{5} \mathrm{O}_{12}$ 陶瓷样品, 样品达到最佳透 过率后，再提高烧结温度，尽管陶瓷样品密度提高, 但其红外透过率却呈下降趋势。

对不同 $\mathrm{Bi}^{3+}$ 掺杂浓度下 $\mathrm{Bi}_{x} \mathrm{Y}_{1-x} \mathrm{Fe}_{5} \mathrm{O}_{12}$ 陶瓷样品 的最佳红外透过率曲线进行比较(见图 3), 发现烧 结的陶瓷样品均具有良好的红外透过率, 尤其是 $x=0.3$ 的陶瓷样品与 $x=0$ 的陶瓷样品的红外透过率 相当，在 $1.0 \sim 10.0 \mu \mathrm{m}$ 波长范围内透过率均超过 $\sim 40 \%$ 。而随着 $\mathrm{Bi}^{3+}$ 掺杂量进一步增加，陶瓷样品的 红外透过率反而降低, 大量 $\mathrm{Bi}^{3+}$ 掺杂的陶瓷样品透 过率明显降低。下文将围绕表 1 所列的 $\mathrm{Bi}^{3+}$ 掺杂量 不同但具有最佳红外透过率的 4 个陶瓷样品进一步 深入研究和讨论分析。

\section{3 相结构分析}

图 4 为 $\mathrm{Bi}_{x} \mathrm{Y}_{1-x} \mathrm{Fe}_{5} \mathrm{O}_{12}(x=0 、 0.3 、 0.6 、 0.9)$ 陶瓷 样品 XRD 谱图。图 4(a)中给出了 YIG 标准粉末衍 射图谱 ${ }^{[24]}$ (JCPDS 43-0507)。通过与 YIG 标准图谱进 行比较, 所有样品的衍射峰与立方 YIG 的衍射峰匹 配良好, 说明测试样品均为石榴石结构, 所有样品 均未发现明显的第二相。图 4(b)为 $2 \theta=68^{\circ} \sim 70^{\circ}$ 的局 部放大 XRD 图谱。观察(842)晶面的衍射峰，可以发 现随着铋掺杂量的增加, 样品的 XRD 衍射峰向低 角度移动, 表明 $\mathrm{Bi}_{x} \mathrm{Y}_{1-x} \mathrm{Fe}_{5} \mathrm{O}_{12}$ 陶瓷的晶格参数随着 铋掺杂量的增加而增大。 $\mathrm{Bi}^{3+}$ 的半径 $(0.1170 \mathrm{~nm})$ 大 于 $\mathrm{Y}^{3+}$ 的半径 $(0.1019 \mathrm{~nm})^{[25]}$, 大半径 $\mathrm{Bi}^{3+}$ 离子通过

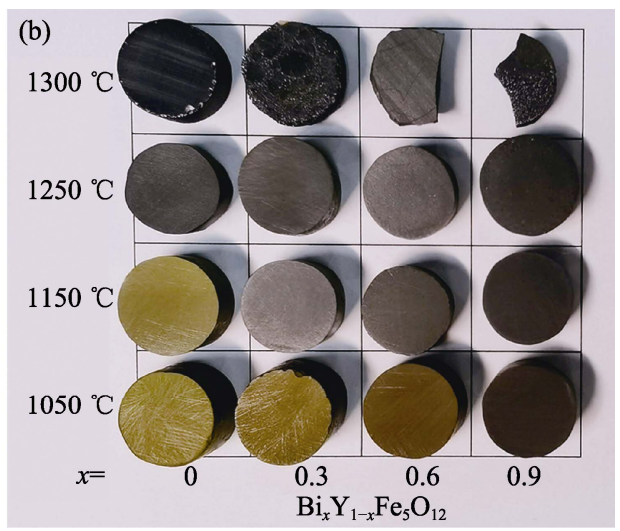

图 $1 \mathrm{Bi}_{x} \mathrm{Y}_{1-x} \mathrm{Fe}_{5} \mathrm{O}_{12}$ 陶瓷样品密度与烧结温度的关系曲线(a)和陶瓷样品实物照片 (b)

Fig. 1 Density of $\mathrm{Bi}_{x} \mathrm{Y}_{1-x} \mathrm{Fe}_{5} \mathrm{O}_{12}$ ceramic samples varies with sintering temperatures (a) and photos of $\mathrm{Bi}_{x} \mathrm{Y}_{1-x} \mathrm{Fe}_{5} \mathrm{O}_{12}$ samples (b) 


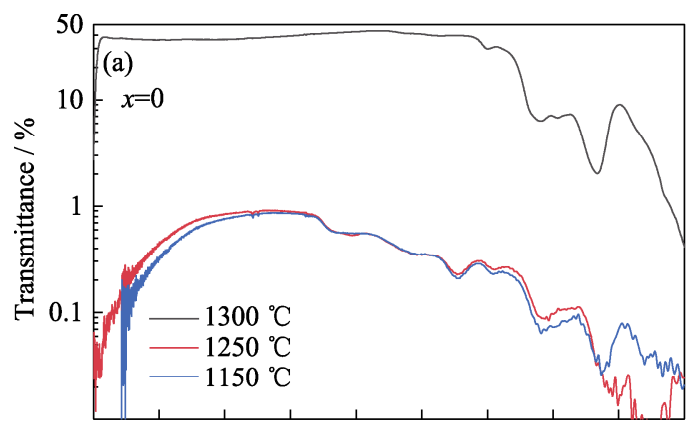

10002000300040005000600070008000900010000 Wavelength / nm

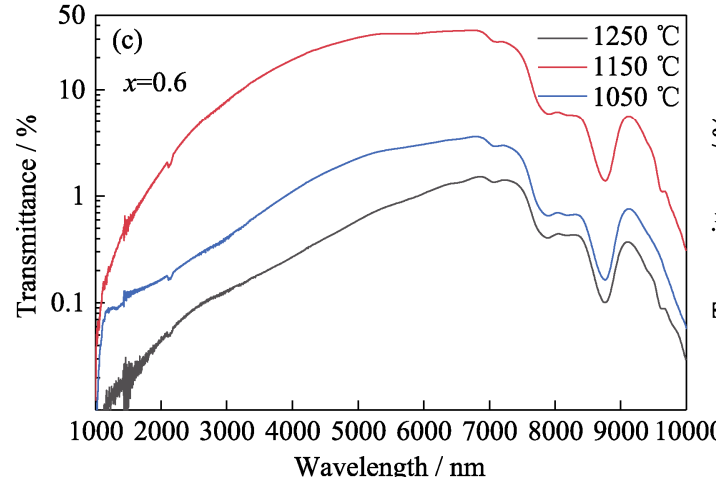

Wavelength / nm

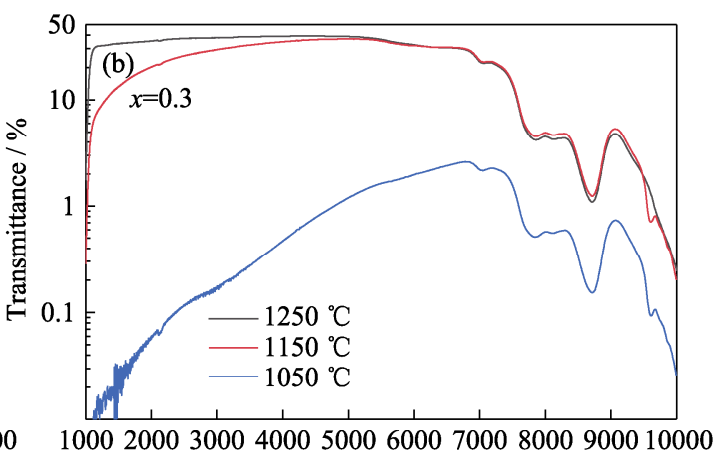

Wavelength / nm

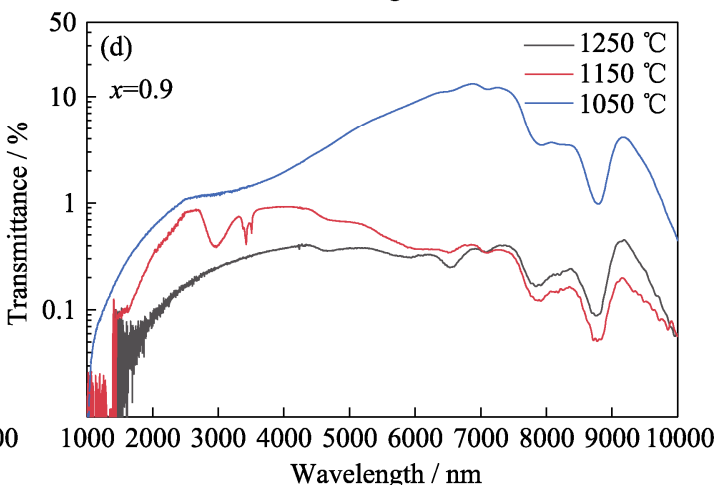

图 2 不同烧结温度 $\mathrm{Bi}_{x} \mathrm{Y}_{1-x} \mathrm{Fe}_{5} \mathrm{O}_{12}$ 陶瓷样品的红外透过率曲线 $(d=0.4 \mathrm{~mm})$

Fig. 2 Infrared transmittance curves of $\mathrm{Bi}_{x} \mathrm{Y}_{1-x} \mathrm{Fe}_{5} \mathrm{O}_{12}$ samples sintered at different temperatures $(d=0.4 \mathrm{~mm})$ Colorful figures are available on website

\section{表 1 最高密度陶瓷样品及最佳透过率陶瓷样品的烧结温度}

Table 1 Sintering temperatures of the samples with the highest density and the highest transmittance

\begin{tabular}{ccc}
\hline & $\begin{array}{c}\text { Sintering temperature } \\
\text { of the sample } \\
\text { with the highest } \\
\text { density } /{ }^{\circ} \mathrm{C}\end{array}$ & $\begin{array}{c}\text { Sintering temperature } \\
\text { of the sample } \\
\text { with the highest } \\
\text { transmittance } /{ }^{\circ} \mathrm{C}\end{array}$ \\
\hline$x=0$ & 1300 & 1300 \\
$x=0.3$ & 1250 & 1250 \\
$x=0.6$ & 1250 & 1150 \\
$x=0.9$ & 1150 & 1050 \\
\hline
\end{tabular}

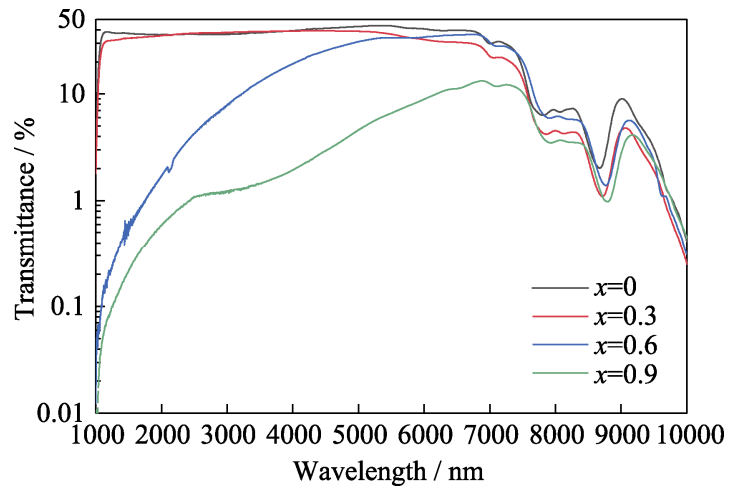

图 $3 \mathrm{Bi}^{3+}$ 掺杂 $\mathrm{Bi}_{x} \mathrm{Y}_{1-x} \mathrm{Fe}_{5} \mathrm{O}_{12}$ 陶瓷样品 $(d=0.4 \mathrm{~mm})$ 的最佳红 外透过率曲线

Fig. 3 Best infrared transmittance curves of $\mathrm{Bi}_{x} \mathrm{Y}_{1-x} \mathrm{Fe}_{5} \mathrm{O}_{12}$ samples with $\mathrm{Bi}^{3+}$ doping $(d=0.4 \mathrm{~mm})$

Colorful figures are available on website
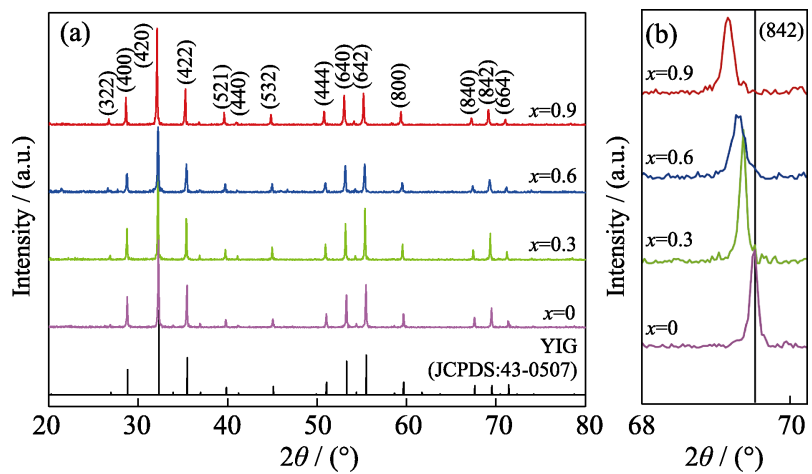

图 $4 \mathrm{Bi}_{x} \mathrm{Y}_{1-x} \mathrm{Fe}_{5} \mathrm{O}_{12}(x=0 、 0.3 、 0.6 、 0.9)$ 样品的 XRD 图谱(a) 及 XRD 谱图中 $2 \theta$ 在 $68^{\circ} \sim 70^{\circ}$ 细节图谱(b)

Fig. 4 XRD patterns of $\mathrm{Bi}_{x} \mathrm{Y}_{1-x} \mathrm{Fe}_{5} \mathrm{O}_{12}(x=0,0.3,0.6,0.9)$ samples (a) and the detail map at $2 \theta=68^{\circ}-70^{\circ}$ (b)

置换十二面体位亚晶格中 $\mathrm{Y}^{3+}$ 离子进入 $\mathrm{YIG}$ 晶胞中, 且始终保持石榴石结构, 导致晶格轻微畸变, 晶面 间距增大。

\section{4 微观形貌表征及元素分析}

图 5 是 $\mathrm{Bi}_{x} \mathrm{Y}_{1-x} \mathrm{Fe}_{5} \mathrm{O}_{12}(x=0 、 0.3 、 0.6 、 0.9)$ 陶瓷 样品的断面显微结构照片。采用截距法测量陶瓷样 品的平均晶粒尺寸, $x=0 、 0.3 、 0.6$ 和 0.9 的陶瓷样品 平均晶粒尺寸分别为 $1.82 、 3.62 、 2.54$ 和 $3.21 \mu \mathrm{m}$ 。 掺入 $\mathrm{Bi}^{3+}$ 导致陶瓷样品的晶粒尺寸不同程度地增大, 这可能由于 $\mathrm{Bi}_{2} \mathrm{O}_{3}$ 的熔点 $\left(825{ }^{\circ} \mathrm{C}\right)$ 较低, 在烧结过程 中起到一定的烧结助剂作用, 不仅降低了 YIG 陶瓷 


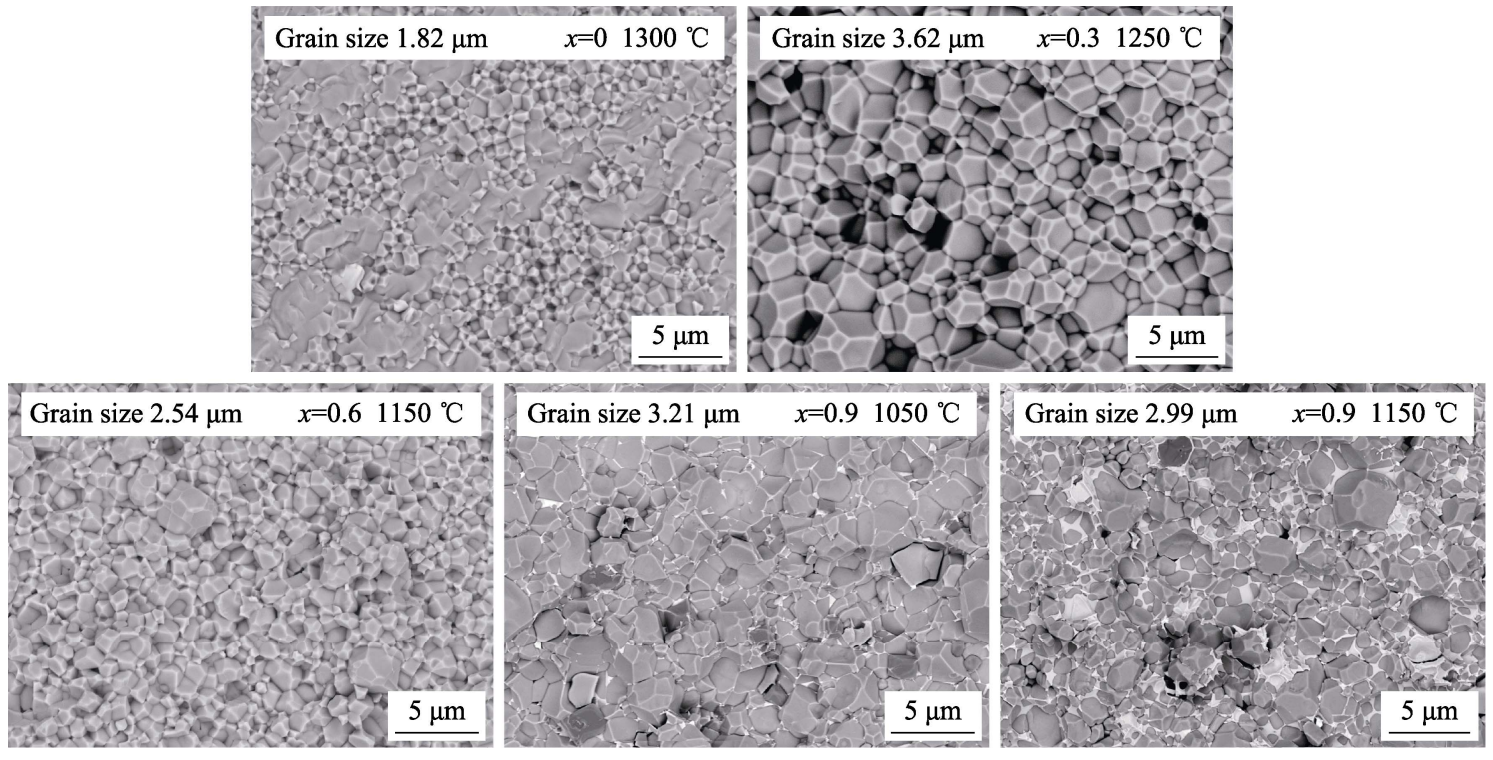

图 $5 \mathrm{Bi}_{x} \mathrm{Y}_{1-x} \mathrm{Fe}_{5} \mathrm{O}_{12}$ 陶瓷样品的断面 SEM 照片

Fig. 5 Cross-section SEM images of the $\mathrm{Bi}_{x} \mathrm{Y}_{1-x} \mathrm{Fe}_{5} \mathrm{O}_{12}$ ceramic samples

的烧结温度, 同时有利于晶界运动, 促进晶粒生长, 使陶瓷晶粒尺寸增大，但因烧结温度不同，平均晶 粒尺寸增大的程度并不一样。 $\mathrm{Bi}_{x} \mathrm{Y}_{1-x} \mathrm{Fe}_{5} \mathrm{O}_{12}(x=0$ 、 $0.3 、 0.6 、 0.9)$ 陶瓷中透过率最佳的样品烧结温度分 别是 1300、1250、1150 和 $1050{ }^{\circ} \mathrm{C}$ ，通过对比它们 的断面 SEM 照片可以看出, 随着掺杂量增加至 $x=0.9$, 晶界处明显出现第二相。第二相的产生可能 是陶瓷样品的透过率随着铋掺杂量的增加而降低的 原因之一。比较 1050 和 $1150{ }^{\circ} \mathrm{C}$ 温度烧结的 $\mathrm{Bi}_{0.9} \mathrm{Y}_{2.1} \mathrm{Fe}_{5} \mathrm{O}_{12}$ 陶瓷样品的断面 SEM 照片, 发现随 着烧结温度提高陶瓷断面晶界处的第二相数量越多 且尺度更大。

通过 EDS 分析 $\mathrm{Bi}_{0.9} \mathrm{Y}_{2.1} \mathrm{Fe}_{5} \mathrm{O}_{12}$ 陶瓷样品中晶粒 与第二相的化学组成, 结果见图 6。白色部分即第二 相(图 6 中点 B)中主要化学元素含量为 Bi: $31.97 \%$, O: $58.72 \%, \mathrm{Fe}: 6.38 \%$ 和 Y: $2.93 \%$ (原子分数), 远远 偏离陶瓷主晶粒的化学组成(图6中点 A), 形成的第 二相主要为氧化铋。分析认为 $\mathrm{Bi}^{3+}$ 离子半径比较大, 大量的 $\mathrm{Bi}^{3+}$ 很难完全固溶在石榴石结构的十二面体 亚晶格中。未固溶进晶格的 $\mathrm{Bi}^{3+}$ 离子, 在晶界处富 集为铋的氧化物, 从而形成第二相。而且铋属于重 金属元素, 其氧化物密度较高, 所以第二相氧化铋 的形成既造成陶瓷样品透过率下降, 又使样品密度 升高。这也解释了提高 $\mathrm{Bi}^{3+}$ 掺杂量后最致密陶瓷烧 结温度与透过率最佳烧结温度不一致的现象。

\section{5 磁性能与磁光性能分析}

图 7(b)为 $\mathrm{Bi}_{x} \mathrm{Y}_{1-x} \mathrm{Fe}_{5} \mathrm{O}_{12}$ 陶瓷烧结样品的磁滞回 线, 表 2 是磁滞回线中相应参数的汇总。结果表明, 随着铋掺杂量增加, 剩余磁化强度和矫顽场强明显
降低, 这有利于降低材料的磁损耗。所有样品在 $0.3 \mathrm{~T}$ 的外磁场下均达到饱和磁化强度, 随着 $\mathrm{Bi}^{3+}$ 离 子掺杂量增加，材料的饱和磁化强度略有增大，而 当 $x=0.9$ 时, 饱和磁化强度降低, 这可能与陶瓷中晶 界处形成的第二相有关。

图 7(a)为法拉第旋转角测试设备示意图, 通过 调节电磁铁电流大小来改变磁场大小。图 7(b)中所 有样品在 $0.3 \mathrm{~T}$ 的外磁场下均达到饱和磁化强度, 故在 $0.3 \mathrm{~T}$ 磁场下测量并计算陶瓷样品的单位长度 旋光角 $\theta_{\mathrm{F}}$ 。通过高斯计探测样品所处位置磁场实际 大小, 确保外磁场均在 $0.3 \sim 0.35 \mathrm{~T}$ 范围内, 以平行 入射 $1064 \mathrm{~nm}$ 及 $1550 \mathrm{~nm}$ 激光为测试光源。

图 7(c)为 $\mathrm{Bi}_{x} \mathrm{Y}_{3-x} \mathrm{Fe}_{5} \mathrm{O}_{12}$ 陶瓷在 1064 和 $1550 \mathrm{~nm}$ 波长下测得的 $\theta_{\mathrm{F}}$ 结果。 $1064 \mathrm{~nm}$ 波长下, 当 $\mathrm{Bi}^{3+}$ 掺 杂量 $x$ 分别为 $0 、 0.3 、 0.6$ 时, $\theta_{\mathrm{F}}$ 测试值分别为 277.6、 -194.2 和 $-703.3\left({ }^{\circ}\right) / \mathrm{cm} ; 1550 \mathrm{~nm}$ 波长下, 当 $\mathrm{Bi}^{3+}$ 掺 杂量 $x$ 分别为 $0 、 0.3 、 0.6 、 0.9$ 时, $\theta_{\mathrm{F}}$ 测试值分别为 $172.0 、-144.6 、-461.5$ 和 $-729.2\left(^{\circ}\right) / \mathrm{cm}$ 。在 $1064 \mathrm{~nm}$ 及 $1550 \mathrm{~nm}$ 波长下，陶瓷材料的法拉第旋转角随着 $\mathrm{Bi}^{3+}$ 掺杂量增加呈反向线性变化, 当 $\mathrm{Bi}^{3+}$ 掺杂量每 增加 $1 \%$ (原子分数)时, $1064 \mathrm{~nm}$ 及 $1550 \mathrm{~nm}$ 波长下 旋转角变化量分别约为 -49.0 和 $-30.2\left(^{\circ}\right) / \mathrm{cm}$ 。研究 结果表明, YIG 陶瓷的磁光性能随 $\mathrm{Bi}^{3+}$ 掺杂量增加 明显增强。

\section{3 结论}

以 $\mathrm{Bi}_{2} \mathrm{O}_{3} 、 \mathrm{Y}_{2} \mathrm{O}_{3}$ 和 $\mathrm{Fe}_{2} \mathrm{O}_{3}$ 粉体为原料, 采用热压 烧结工艺制备了铋掺杂 YIG 多晶陶瓷。确定了不同 

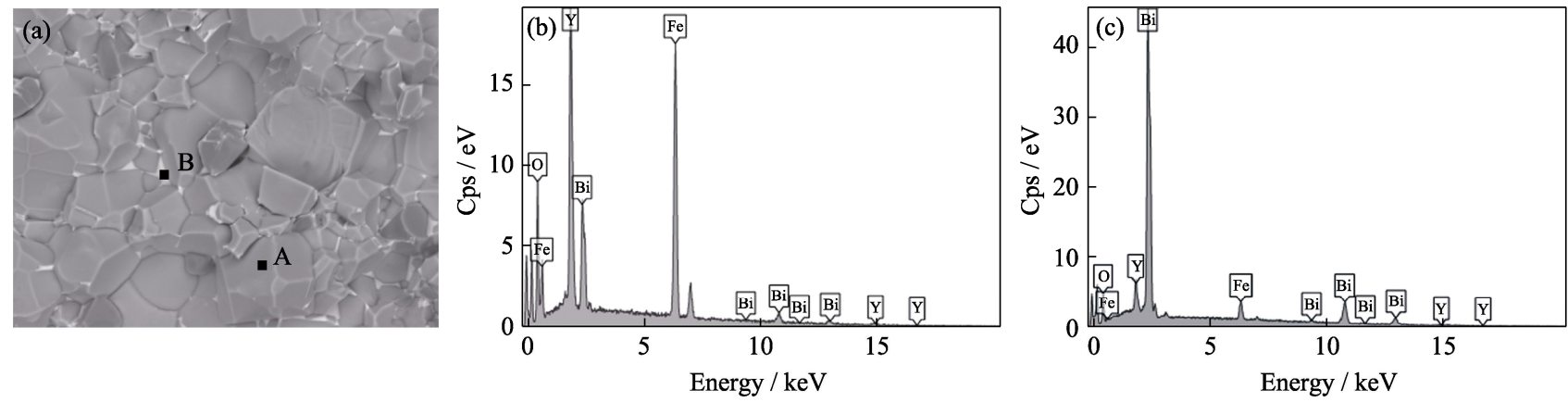

图 $61050{ }^{\circ} \mathrm{C}$ 下烧结 $\mathrm{Bi}_{0.9} \mathrm{Y}_{2.1} \mathrm{Fe}_{5} \mathrm{O}_{12}$ 陶瓷的 SEM 照片(a)和 EDS 谱图(b, c)

Fig. 6 SEM image (a) and EDS patterns of the $\mathrm{Bi}_{0.9} \mathrm{Y}_{2.1} \mathrm{Fe}_{5} \mathrm{O}_{12}$ ceramics sintered at $1050{ }^{\circ} \mathrm{C}$ (b, c c) corresponding to the site of $\mathrm{A}$ and $\mathrm{B}$ in (a), respectively

(a) Light Polarizer
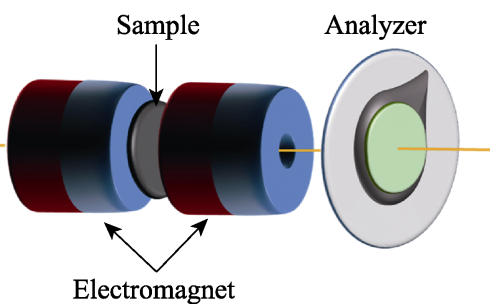

Detector

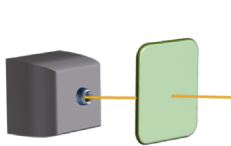

Electromagnet

$0.98 \mathrm{~mW}$
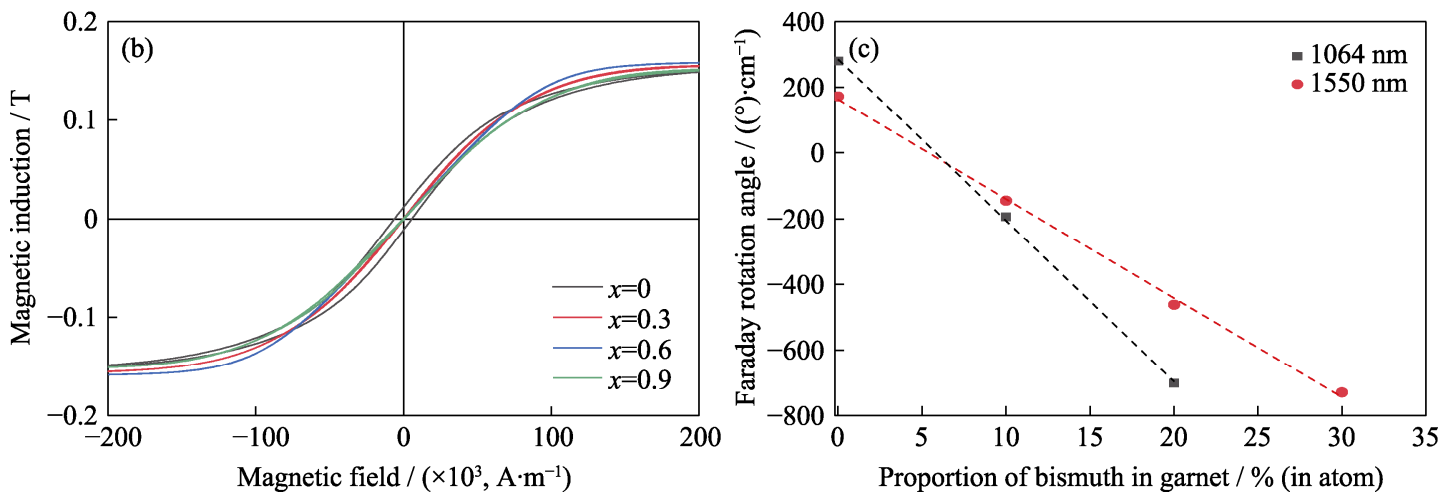

图 7 法拉第旋转角测试设备示意图(a), $\mathrm{Bi}_{x} \mathrm{Y}_{1-x} \mathrm{Fe}_{5} \mathrm{O}_{12}$ 陶瓷样品的磁滞回线(b),

以及陶瓷样品在 1064 和 $1550 \mathrm{~nm}$ 处的 $\theta_{\mathrm{F}}$ 与铋的相对含量的关系(c)

Fig. 7 Schematic diagram of Faraday rotation angle test equipment (a), hysteresis loops (b) of $\mathrm{Bi}_{x} \mathrm{Y}_{1-x} \mathrm{Fe}_{5} \mathrm{O}_{12}$ samples, and Faraday rotation angle of the ceramic sample varies with the proportion of bismuth at 1064 and $1550 \mathrm{~nm}(\mathrm{c})$ Colorful figures are available on website

表 2 不同 $\mathbf{B i}^{3+}$ 掺杂量 YIG 陶瓷样品的磁性能参数 Table 2 Magnetic performance parameters of the sintered $\mathrm{Bi}_{x} \mathrm{Y}_{1-x} \mathrm{Fe}_{5} \mathrm{O}_{12}$ samples with different amounts of $\mathrm{Bi}^{3+}$ doping

\begin{tabular}{lccc}
\hline$x$ & $\begin{array}{c}\text { Residual } \\
\text { magnetization, } \\
4 \pi M_{\mathrm{r}} / \mathrm{T}\end{array}$ & $\begin{array}{c}\text { Saturation } \\
\text { magnetization, } \\
4 \pi M_{\mathrm{s}} / \mathrm{T}\end{array}$ & $\begin{array}{c}\text { Coercive } \\
\text { field, } H_{\mathrm{c}} / \\
\left(\mathrm{A} \cdot \mathrm{m}^{-1}\right)\end{array}$ \\
\hline 0 & $1.32 \times 10^{-2}$ & 0.1543 & 6029.6 \\
0.3 & $6.59 \times 10^{-3}$ & 0.1577 & 369.2 \\
0.6 & $5.02 \times 10^{-4}$ & 0.1596 & 310.4 \\
0.9 & $2.86 \times 10^{-4}$ & 0.1536 & 179.8 \\
\hline
\end{tabular}

铋掺杂量下的最佳烧结温度, 验证了掺杂铋有利于 多晶 YIG 陶瓷烧结, 发现掺杂适量铋使 YIG 陶瓷样 品呈现出优异的磁光性能, 但掺杂过多铋易导致材

\section{料光学透过性下降。}

1)通过固相反应和热压烧结制备了光学性能优 异的 $\mathrm{Bi}^{3+}$ 掺杂 $\mathrm{YIG}$ 多晶陶瓷, 并且随着 $\mathrm{Bi}^{3+}$ 掺杂量 增大，陶瓷烧结温度显著降低。

2)掺杂大量 $\mathrm{Bi}^{3+}$ 时，部分 $\mathrm{Bi}^{3+}$ 不能固溶进晶格, 富余的 $\mathrm{Bi}^{3+}$ 富集在晶界处形成第二相, 导致陶瓷红 外透过率降低及密度异常变化。

3)磁滞回线测试结果显示, 铋掺杂使 YIG 陶瓷 的剩余磁化强度和矫顽场强明显降低, 有利于材料 的磁损耗降低, 优化了材料磁性能。

4) $\mathrm{Bi}^{3+}$ 掺杂使 YIG 陶瓷的磁光性能明显增强, $\mathrm{Bi}^{3+}$ 掺杂量每增加 $1 \%$ (原子分数), 1064 及 $1550 \mathrm{~nm}$ 波 
长下陶瓷样品的法拉第旋转角变化量分别约为 -49.0 和 $-30.2\left(^{\circ}\right) / \mathrm{cm}$ 。 $\mathrm{Bi}_{0.6} \mathrm{Y}_{2.4} \mathrm{Fe}_{5} \mathrm{O}_{12}$ 陶瓷在 1064 和 $1550 \mathrm{~nm}$ 波长下, $\theta_{\mathrm{F}}$ 分别达到 -703.3 和 $-461.5\left(^{\circ}\right) / \mathrm{cm}$, $\mathrm{Bi}_{0.9} \mathrm{Y}_{2.1} \mathrm{Fe}_{5} \mathrm{O}_{12}$ 陶瓷在 $1550 \mathrm{~nm}$ 波长下, $\theta_{\mathrm{F}}$ 为 $-729.2\left(^{\circ}\right) / \mathrm{cm}$, 均远高于未掺杂 YIG 陶瓷。

\section{参考文献:}

[1] SNETKOV I, PALASHOV O. Faraday isolator based on a TSAG single crystal with compensation of thermally induced depolarization inside magnetic field. Optical Materials, 2015, 42: 293-297.

[2] Pelenovich V O, VAlieV U V, ZHOU L, et al. Magnetooptical spectrometer based on photoelastic modulator with optical feedback and its application in study of $f$-electron materials. Optical Materials, 2016, 55: 115-120.

[3] WANG Y, ZHANG L, ZHUO Z, et al. All-normal dispersion fiber lasers with magneto-optical polarization controllers. Applied Optics, 2017, 56(3): 404-408.

[4] CRASSEE I, LEVALLOIS J, WALTER A L, et al. Giant Faraday rotation in single and multilayer graphene. Nature Physics, 2011, 7(1): 48-51

[5] LECRAW R C, WOOD D L, DILLON J F, et al. The optical transparency of yttrium iron garnet in the near Infrared. Applied Physics Letters, 1965, 7(1): 27-28.

[6] DURCOK S, POLLERT E, SIMSA Z, et al. Growth of YIG and BiGdIG single crystals for magnetooptical applications. Materials Chemistry \& Physics, 1996, 45(2): 124-129.

[7] GOTO H, MAEDA I, NAKANO T, et al. An evaluation of the magneto-optical characteristics of YIG single crystals. Journal of Magnetism \& Magnetic Materials, 1983, 31: 779-780.

[8] LIM H J, DE MATTEI R C, FEIGELSON R S. Growth of single crystal YIG fibers by the laser heated pedestal growth method. MRS Online Proceedings Library (OPL), 1997, 481(1): 83-88.

[9] JIANG Y, SHEN H, XU J Y, et al. Large size rare earth iron garnet single crystals grown by the flux-Bridgman method. Journal of Rare Earths, 2021, 39(12): 1547-1553.

[10] JIN W, GAI L, LI C, et al. Crystal growth and characterization of $\mathrm{Ce}_{x} \mathrm{Y}_{3-x} \mathrm{Fe}_{5} \mathrm{O}_{12}$ single crystal by optical floating zone method. Physica B Condensed Matter, 2020, 588: 412168.

[11] IKESUE A, AUNG Y L. Development of optical grade polycrystalline YIG ceramics for Faraday rotator. Journal of the American Ceramic Society, 2018, 101(11): 5120-5126.

[12] XU H, YANG H. Magnetic properties of Ce, Dy-substituted yttrium iron garnet ferrite powders fabricated using a Sol-Gel method.
Physica Status Solidi (a), 2007, 204(4): 1203-1209.

[13] WANG J Q, YANG J, JIN Y L, et al. Effect of manganese addition on the microstructure and electromagnetic properties of YIG. Journal of Rare Earths (English Edition), 2011, 29(6): 562-566.

[14] TABOR W J, ANDERSON A W, VANUITERT L G. Visible and infrared Faraday rotation and birefringence of single-crystal rareearth orthoferrites. Journal of Applied Physics, 1970, 41(7): 3018-3021.

[15] COOPER R W, DILlON J F, VANUITERT L G, et al. Magnetooptic light modulators. Radio and Electronic Engineer, 1970, 39(6): 302-304.

[16] KAHN F J, PERSHAN P S, REMEIKA J P. Ultraviolet magnetooptical properties of single-crystal ortho-ferrites, garnets, and other ferric oxide compounds. Physical Review, 1969, 186(3): 891.

[17] FECHINE P B A, MORETZSOHN R S T, COSTA R C S, et al. Magneto-dielectric properties of the $\mathrm{Y}_{3} \mathrm{Fe}_{5} \mathrm{O}_{12}$ and $\mathrm{Gd}_{3} \mathrm{Fe}_{5} \mathrm{O}_{12}$ dielectric ferrite resonator antennas. Microwave and Optical Technology Letters, 2008, 50(11): 2852-2857.

[18] AUNG Y L, IKESUE A, WATANABE T, et al. Bi substituted YIG ceramics isolator for optical communication. Journal of Alloys \& Compounds, 2019, 811: 152059

[19] SCOTT G B, LACKLISON D E, PAGE J L. The effect of octahedral $\mathrm{Fe}^{3+}$ and tetrahedral $\mathrm{Fe}^{3+}$ dilution on the Faraday spectra of bismuth-doped iron garnets. Journal of Physics C: Solid State Physics, 1975, 8(4): 519-529.

[20] DESCHANVRES J L, LANGLET M, BOCHU B, et al. Growth of Bi-substituted YIG thin films for magneto-optic applications. Journal of Magnetism and Magnetic Materials, 1991, 101(1): 224-226.

[21] ALLEN G A, DIONNE G F. Application of permittivity tensor for accurate interpretation of magneto-optical spectra. Journal of Applied Physics, 1993, 73(10): 6130-6132

[22] ISHIBASHI T, LOU G, MEGURO A, et al. Magneto-optical imaging plate using bismuth-substituted iron garnet film prepared by metal-organic decomposition. Sensors and materials: An International Journal on Sensor Technology, 2015, 27(10): 965-970.

[23] ZHANG G, XU X, CHONG T. Faraday rotation spectra of bismuth-substituted rare-earth iron garnet crystals in optical communication band. Journal of Applied Physics, 2004, 95(10): 5267-5270.

[24] YANG Y, LI X, LIU Z, et al. Pressureless sintering of YIG ceramics from coprecipitated nano-powders. Magnetochemistry, 2021, 7(5): 56.

[25] NIYAIFAR M, MOHAMMADPOUR H. Study on magnetic role of $\mathrm{Bi}^{3+}$ ion by random cation distribution model in Bi-YIG system. Journal of Magnetism and Magnetic Materials, 2015, 100(396): $65-70$. 